\title{
28 Research Square \\ Effects of Integrative Medicine on The Post-Stroke Cognitive Impairment (PSCI) Patients: A Study Protocol for A Randomized Multicentric Controlled
}

Trial

\section{Xinyun Li}

Zhejiang Chinese Medical University, Hangzhou, CN; https://orcid.org/0000-0002-2793-3367

\section{Kelin He}

Zhejiang Chinese Medical University

\section{Lei Wu}

Third Affiliated Hospital of Zhejiang Chinese Medical university

\section{Weijun Zheng}

Zhejiang Chinese Medical University

\section{Kaitao Luo}

Jiaxing hospital of Chinese medcine

Liping Li

Hangzhou Hospital of Traditional Chinese Medicine

\section{Daowei Zan}

Jiaxing hospital of Chinese medcine

\section{Fengjia $\mathrm{Ni}$}

Zhejiang Chinese Medical University

\section{Ruijie Ma ( $\square$ maria7878@sina.com )}

Zhejiang Chinese Medical University

\section{Fengjia Ni}

Jiaxing Chinese Medical Hospital

\section{Study protocol}

Keywords: acupuncture, herbs, stroke, cognitive, RCT

Posted Date: June 28th, 2021

DOl: https://doi.org/10.21203/rs.3.rs-424939/v1

License: (c) (i) This work is licensed under a Creative Commons Attribution 4.0 International License. Read Full License 
Page $2 / 18$ 


\section{Abstract \\ Background}

Due to limitation of rehabilitation in cognitive impairment after stroke, as the main parts of traditional Chinese medicine, acupuncture and herbs have their advantage effect in clinical practice for post-stroke cognitive impairment (PSCl). Therefore, forming a standardized and propagable TCM project of PSCI becomes necessary. This trail will solve the above problem.

\section{Methods}

In total, 126 stroke patients will be recruited from the inpatient and outpatient departments of The Third Affiliated Hospital of Zhejiang Chinese Medical University (Hangzhou, China), Jiaxing Hospital of Traditional Chinese Medicine and Hangzhou Hospital of Traditional Chinese Medicine to participate in this study with randomization. All participants will continue to receive basic treatment and cognitive function regular training, while successive 12 weeks of acupuncture and herbs will be provided to patients assigned to the integrative medicine group. Montreal cognitive assessment (MOCA), mini-mental state examination, activity of daily living (ADL) score, hamilton depression scale (HAMD), functional nearinfrared spectroscopy (fNIRS) and RESTing-state functional MRI (RS-fmri) will be used to measure the outcomes.

\section{Discussion}

This trial may provide high-quality RCT evidence regarding the clinical effectiveness and safety of acupuncture and herbs for PSCI patient.

\section{Trial registration:}

ClinicalTrials.gov (ID: NCT 04596072)

\section{Background}

In recent years, stroke has been recognized by the Global Burden of Disease (GBD) as the second major factor affecting disability and loss of healthy life years, videlicet, stroke is the second leading cause of death and disability in the world[1]. A long-term outcome in stroke patients with cognitive impairment clinical study has found that stroke without cognitive impairment is more likely to live longer than with cognitive impairment[2]. Due to abnormalities of the visual spatial function and executive function, structure, memory, orientation, attention in PSCI patients, their daily life and social activity ability are greatly affected, and heavy burden is pressed to the society and family[3]. 
Currently, the rehabilitation of PSCl includes drug therapy and non-drug therapy[4]. There is no specific effective drug for the treatment of PSCl, but considering that the vascular dementia and dementia have certain overlaps in neuropathology and neurochemistry mechanism, cholinesterase inhibitors and non competitive $\mathrm{N}$ - methyl -D- aspartic acid receptor antagonist have been approved for improving cognitive function after stroke[4]. In addition, new drugs have been developed but not commonly used, such as neurotrophic enhancers like Bryostatin[5]. Other neurotransmitters are also used in clinic, but they are lack of efficiency study on PCSI[6]. The non-drug therapy includes cognitive training therapy, occupational therapy, music therapy[7], repetitive transcranial magnetic stimulation (rTMS) [8], transcranial electrical stimulation[9], psychological intervention[10] and other rehabilitation methods. However, there is still lacking for large sample clinical studies to verify their clinical efficacy and safety of non-drug therapy.

Nowadays, traditional Chinese medicine (TCM) has become an important therapy of PSCl treatment due to its advantages such as low cost, simple manipulation and favorable effectiveness[11]. For TCM clinical application, acupuncture and moxibustion, herbs and Qigong have achieved excellent results in $\mathrm{PSCl}$ treatment [12-17]. Although many clinical studies have shown that TCM treatment of PSCI is effective. Hence, a multi-center, large sample and randomized controlled study method is designed to assess the exact efficacy of acupuncture and herbs in the treatment of $\mathrm{PSCl}$, so as to improve the evidence level of TCM application in the clinical treatment and form a standardized and propagable TCM project.

\section{Methods/design}

\section{Objectives}

The purpose of this study is to evaluate the efficacy and safety of acupuncture combined herbs treating post-stroke cognitive impairment by comparing changes in cognitive assessment, activity of daily living $\rrbracket$ depressive state and the changes of brain regions after treatment. This study is expected to develop a standard, systematic and safe TCM integrative treatment plan for cognitive impairment after stroke, and improve the quality of life of patients.

\section{Trail design}

126 patients treated in the Third Affiliated Hospital of Zhejiang Chinese Medical University, Jiaxing Hospital of Traditional Chinese Medicine, Hangzhou Hospital of Traditional Chinese Medicine will be recruited from April 2019 to December 2023. The study plan has got approved by the ethics committee of the three hospitals. The clinical trial will be conducted in accordance with the 2010 Consolidated Standards of Reporting Trials (CONSORT) guidelines[18, 19] and the Standards for Reporting Interventions in Controlled Trials of Acupuncture (STRICTA) [20]. The 126 patients will be distributed to the control group and the TCM group in a 1:1 ratio. The control group will receive basic treatment and cognitive function regular training for 12 weeks, while the TCM group will receive basic treatment, cognitive function regular training, acupuncture and herbs for 12 weeks. The results will be measured by Montreal Cognitive Assessment (MOCA), mini-Mental State Examination, Activity of Daily Living (ADL) 
score, Hamilton Depression Scale (HAMD), Functional near-infrared spectroscopy (fNIRS) and RESTingstate functional MRI (RS-fmri). Fig 1 shows the trial design.

\section{Participants recruitment}

Patients admitted to the Acupuncture and Moxibustion Department, Neurology Department and Rehabilitation Department of the Third Affiliated Hospital of Zhejiang Chinese Medical University, Rehabilitation Department of Jiaxing Hospital of Traditional Chinese Medicine, and Rehabilitation Department of Hangzhou Hospital of Traditional Chinese Medicine. The public obtain the message of this study by each hospital's WeChat Official Account, local newspapers, hospital's roll up banners and brochure. Interested patients can contact the investigator through phone, message, email, or WeChat.

\section{Inclusion criteria}

The patients should meet all the following criteria, which will be screened by a neurologist:

(1) Diagnosis of stroke in traditional Chinese medicine[21] and PSCI [22];

(2) Age above 18;

(3) The course of disease ranges from 30 to 180 days;

(4) The patient's legal guardian signs the informed consent;

(5) Montreal Cognitive Assessment (MoCA) score is 17-26, and if the duration of patient's education is less than or equal to 12 years, one score will be deducted;

(6) Hamilton Depression Scale (HAMD) score <20;

(7) Patient meets the requirements for indications of acupuncture and moxibustion techniques, and volunteers to accept acupuncture treatment without serious complications.

\section{Exclusion criteria}

The patients who meet the following criteria should be excluded and it will be screened by a neurologist:

(1) Cognitive impairment caused by subarachnoid hemorrhage, transient ischemic attack, or other intracranial lesions such as intracranial tumors, aneurysms, vascular malformations, cysticercosis, schistosomiasis, encephalitis, meningitis, hydrocephalus, sequelae of brain trauma;

(2) non-atherosclerotic thrombotic cerebral infarction (such as cardiac embolism, coagulation state, endovascular shedding, arteritis);

(3) Pregnant or lactating women; 
(4) Patients with severe primary chronic diseases including heart, liver, kidney and other viscera, as well as the endocrine system and hematopoietic system), severe dementia, serious language understanding disorders, mental illness;

(5) Patient with a variety of bleeding tendency diseases;

(6) Patients who do not meet the inclusion criteria and are not suitable for clinical observation.

\section{Interventions}

Both of the two groups will receive basic treatment and cognitive function regular training, which is conducted by experienced doctors and rehabilitation therapists following the Standard Operation Procedure document (SOP) and Chinese Stroke Association guidelines for clinical management of cerebrovascular disorders[23]. During the whole study process, training sessions for investigators will be held regularly so that the equal quality of all the interventions will be guaranteed.

\section{Control group}

The control group will receive basic treatment and cognitive function regular training, including cognitive function training, daily life activity training, psychological support therapy and so on. Treatment will be performed $30 \mathrm{~min}$ each time, and 5 times a week for 12 weeks.

\section{Integrative Medicine group}

The integrative medicine group will receive basic treatment and cognitive function regular training, which are the same as the control group. Besides, acupuncture and herbs are administered 5 times a week for 4 weeks.

\section{Acupuncture}

Acupoints: Shenting (GV24) (bilateral), Benshen (GB13), Baihui (GV20), Sishencong (EX-HN1), Fengchi (GB20) (bilateral), Fenglong (ST40) (bilateral), Zusanli (ST36) (bilateral), Xuanzhong (GB39) (bilateral), Top midline, Forehead midline, No.1 line near the top (bilateral).

Needles: $0.25 \mathrm{~mm} \times 40 \mathrm{~mm}$ stainless steel needle of Huatuo Brand (produced by Suzhou Medical Supplies Factory Co., LTD.).

Manipulation: The patient will lie in prone position, and the acupoints skin will be disinfected with $75 \%$ ethanol. Different manipulations are used for different acupoints (table 1). Acupuncture will be applied five times weekly for 12 weeks, and every treatment lasts $30 \mathrm{~min}$.

\section{Herbs}

The herbal treatment will be based on the syndrome differentiation. All the syndromes and treatments will be divided into the following five types. The herbs will be given five times weekly for 12 weeks, and twice 
for one day.

(1) Liver and kidney yin deficiency syndrome

Treatment: To tonify liver and kidney, fill essence and restore energy.

The formulas: Liu-Wei-Di-Huang-Wan (Six-Ingredient Rehmannia Pill) or Chinese patent medicine with similar efficacy.

(2) Spleen and kidney deficiency syndrome

Treatment: To tonify kidney and spleen, replenish qi to invigorate essence.

The formulas: Gui-Pi-Tang (Spleen-Restoring Decoction) and Huan-Shao-Dan, or Chinese patent medicine with similar efficacy.

(3) Phlegm and turbidity obstruct brain

Treatment: To clear phlegm for resuscitation, invigorate the spleen and transform turbidity.

The formulas: Di-Tan-Tang (Phlegm-Flushing Decoction) or Chinese patent medicine with similar efficacy.

(4) Stagnation of blood stasis syndrome

Treatment: To promote blood circulation to remove blood stasis, awake and enlighten brain

The formulas: Tong-Qiao-Huo-Xue-Tang \Orifice-Opening and Blood-Invigorating Decoction囚or Chinese patent medicine with similar efficacy.

(5) Hyperactivity of liver-yang syndrome

Treatment: To calm liver wind, active blood and clear heat, tonify the liver and kidney.

The formulas: Tian-Ma-Gou-Teng-Yin (Gastrodia and Uncaria Decoction) or Chinese patent medicine with similar efficacy.

\section{Outcome measures}

Table 2 Outcome measurements at each timepoint

\section{Primary outcome measures}

Improvement of Cognitive function: we will use Montreal Cognitive Assessment (MOCA) score from baseline to 12 weeks. This assessment has high sensitivity and specificity of assessing cognitive impairment, and it is evaluated from visual spatial function, language function, image ability, orientation and so on. 


\section{Secondary outcome indicators}

1. Montreal cognitive assessment scale score change trend

The MoCA scores of baseline, 0 week, 8 weeks, 12 weeks, 16 weeks and 24 weeks will be analyzed to evaluate the Montreal cognitive assessment scale score change trend.

2. Changes in memory, attention and computational ability, language function

We will divide MoCA scores of baseline and 12 weeks into memory, attention and computational ability, and language function. Different analyses will be used to evaluate different abilities.

3. The rate of change in the scores of mini-Mental State Examination (MMSE)

MMSE scores will be analyzed at baseline, 0 week, 8 weeks, 12 weeks, 16 weeks and 24 weeks.

4. Changes in Daily Living ability

The Activity of Daily Living (ADL) score will be analyzed at 0 week, 12 weeks, 16 weeks and 24 weeks.

5. Changes in depressive status

Hamilton Depression Scale (HAMD) scores will be analyzed 0 week, 12 weeks, 16 weeks and 24 weeks.

6. Changes in the patterns of related brain regions in cognitive impairment

The changes of amplitude of low-frequency fluctuations (ALFFs), regional homogeneity (ReHo) and functional connecting (FC) of RESTing-state functional MRI (RS-fmri) will be analyzed at baseline and 12 weeks.

7. Neural activity in the brain

The changes of the concentration of oxy-hemoglobin ( $\mathrm{HbO2})$, deoxy-hemoglobin $(\mathrm{Hbb})$, cerebral blood flow (CBF) and cerebral blood volume of Functional near-infrared spectroscopy (fNIRS) will be analyzed at baseline and 12 weeks.

8. Effective rate of cognitive function treatment

MoCA score will be calculated from baseline to 12 weeks of treatment and effective rate of treatment will follow the specific criteria. Recovered: the score increased by $\geq 90 \%$ compared with the baseline. Significantly effective: the score increased between $70 \%$ and $89 \%$ compared with the baseline. Effective: the score increased between $30 \%$ and $69 \%$ compared with the baseline. Ineffective: the score increased < $30 \%$ compared with the baseline.

\section{Sample size}


The calculation of sample size will be based on the change of MoCA scores. Research and clinical experience suggest that in an acupuncture group and a non-acupuncture group, a clinically significant therapeutic effect is defined as a MoCA score difference of 2.6 between [12, 24, 25]. A two-sided, twosample $t$ test at the 0.05 significance level will be used. With an estimated $20 \%$ dropout rate at final follow-up, 63 cases are required for each group, which means 126 patients in total will be enrolled.

\section{Randomization and blinding}

Eligible patients will be randomly assigned (1:1) to either the TCM group or the control group.

Randomisation will be stratified by centers. The permuted block ( 4 or 6 patients per block) randomisation sequence, including stratification, will be prepared by a statistician not involved in the trial using $\mathrm{R}$ (version 4.0.0).

Because it is an open trial, no blind will be required. The acupuncture and herbal treatments will be performed by licensed acupuncturists with a master degree. The efficacy of acupuncture will be evaluated by a RA. Patients who are enrolled will be aware of their group assignment.

\section{Statistical analysis}

No matter the primary or secondary outcomes will be analyzed by using modified intention-to-treat exposed population. In this study, the primary and secondary outcomes will be analyzed by using the modified intention-to-treat analysis. The primary outcome measures will be analyzed using a hybrid effects model (MMRM) designed by repeated measurements. The secondary outcome measures (MMSE, $A D L, H A M D$ ) will be analyzed by means of covariance analysis. The study will carry out statistical description of the adverse events, and compare the differences between groups using Chi-square test or Fisher's exact as an appropriate method.

The results of rs-fMRI and fNIRS will be imputed using multiple imputation methods, and the specialized analysis will be supported by Jia XZ group from Hangzhou Normal University.

\section{Collection and management of data}

The designed case report form (CRF) will collect each participant's data and transfer them into our database. We set up a data collection database to make sure the integrity of the trail, which is established on 0-trial + Clinical Trial Execution management platform V4.0 (o-Trial). The core management system of platform is applied for the registered clinical projects. With mobile internet technology, the experimental trial is able to finish completely, and achieve the steady progress of the trail.

\section{Safety monitoring}

Presently, adverse events (AEs) of acupuncture are few and mild, such as pain, numbness, skin bruising. And herbs may cause allergy, liver and renal dysfunctions. We will record any serious AEs associated with 
the trial, and immediately report to the main researchers and take corresponding measures. If the AEs are too severe, the patients will be withdrawn from the study.

\section{Quality control}

All the intervention and measures will be conducted by experienced physicians and therapists with the professional license. All the physicians and therapists will receive two-day professional training to familiarize with treatment options and training sessions will be held regularly.

To standardize clinical practices and provide clinical quality assurance, a Standard Operation Procedure document (SOP) will be developed to ensure consistency among different investigators. The SOP will include how to screen the participants, how to carry out randomization and blinding, and standardized manipulation procedures. Therefore, the management will be standardized and achievable.

\section{Discussion}

TCM has significant advantages in the treatment of PSCI and its main therapies include acupuncture, herbs, and tuina, all of which are proved effective [26-33]. However, most of the clinical researches are based on personal experience, and there is no unified method to standardize the TCM treatment of PSCI. Moreover, there are few large-scale, multi-center, high-quality RCT studies. This protocol will make up the imperfections.

Nevertheless, there are still some limitations in this study. 1) Acupuncture and herbs may be perceived by patients, thus blinding method is impossible, and the patients of the control group will be suspicious and interfere with treatment. For this purpose, we will require the patients of the two groups not being treated in the same room at the same time, and manipulators, valuator and data processor are needed to be isolated from each other, so it will reduce bias of nonblinding as much as possible. 2) During the fNIRS and fMRI exams, the patients need to keep clam and maintain the head position. Due to some patients with cognitive disorder, they have a poor cooperation degree. Thus, we will assess patient status before exam and repeat exam if the results are wildly inaccurate. Hence, we will complete collection of the follow-up phase in all manner of ways, such as inviting participants to the clinic for further consultation, or visiting them at their homes, or using Internet video chat with them.

\section{Trial Status}

This study is currently in the recruitment phase. The first patient was enrolled in November 2020, and the study is expected to end in December 2023. (The protocol version number is V1.0, the protocol ID: 2020ZJZS002, 11/14/2020)

\section{Declarations}


We thank Professor Zheng WJ and Cheng RC from Zhejiang Chinese Medical University (Hangzhou, China) for randomisation and statistical analysis.

We thank Jia XZ from Hangzhou Normal University for NIRS and fMRI analysis.

We thank the staff of Quanrong (Shanghai) Pharmaceutical Technology Development Co., LTD for their substantial contributions to the data collection.

We acknowledge the help and contributions from the research assistants, acupuncturists, experts and investigators in each center.

We thank the patients with PSCl for participating in this trial.

Contributors $\mathrm{He} \mathrm{KL}$, Wu L participated in the design of the trial, creating the data analysis plan and drafting the manuscript.

Luo KT, Li LP, Zan DW, He KL, Li SW and Ni FJ collected the information needed for the performance of this trial in each center.

All the authors discussed, read and revised the manuscript, and gave final approval for the publication of this study protocol.

\section{Author contributions}

Xinyun Li and Kelin He contributed equally to this work as co-first authors.

Ruijie Ma designed this study.

Funding This study was supported by Traditional Chinese Medicine Modernization Project, ID:2020ZX011, Zhejiang TCM Science and Technology Program, Zhejiang Administration of Traditional Chinese Medicine, China. Zhejiang Chinese Medical University Research Fund, No: 2018ZY17, Zhejiang Chinese Medical University, China. Zhejiang High-level Talents Special Support Program, China. Key TCM disciplines(traditional Chinese medicine encephalopathy) of Zhejiang, China. Key Laboratory of Acupuncture and Neurology of Zhejiang Province, Department of Neurobiology and Acupuncture Research, The Third Clinical Medical College, Zhejiang Chinese Medical University, Hangzhou310053, China.

Competing interests None declared.

Patient consent for publication Not required.

Provenance and peer review Not commissioned; externally peer reviewed.

Data sharing statement The submitted manuscript is a study protocol which includes no primary data now. Further information unaddressed can be obtained from the corresponding author by the contact 
methods provided in the manuscript.

Open access This is an open access article distributed in accordance with the Creative Commons Attribution Non Commercial (CC BY-NC 4.0) license, which permits others to distribute, remix, adapt, build upon this work non-commercially, and license their derivative works on different terms, provided the original work is properly cited, appropriate credit is given, any changes made indicated, and the use is non-commercial. See: http://creativecommons.org/licenses/by-nc/4.0/.

\section{References}

1. Global burden of 369 diseases and injuries in 204 countries and territories, 1990-2019: a systematic analysis for the Global Burden of Disease Study 2019. Lancet (London, England) 2020, 396(10258):1204-1222.

2. Obaid M, Flach C, Marshall I, Wolfe DA, Douiri C. A: Long-Term Outcomes in Stroke Patients with Cognitive Impairment: A Population-Based Study. Geriatrics (Basel, Switzerland) 2020, 5(2).

3. Døhl Ø, Halsteinli V, Askim T, Gunnes M, Ihle-Hansen H, Indredavik B, Langhammer B, Phan A, Magnussen J. Factors contributing to post-stroke health care utilization and costs, secondary results from the life after stroke (LAST) study. BMC Health Serv Res. 2020;20(1):288.

4. Wang Y, Han S, Qin H, Zheng H, Jiang B, Cao Y, Gao Y, Guan L, Jia Q, Jiang Y, et al. Chinese Stroke Association guidelines for clinical management of cerebrovascular disorders: executive summary and 2019 update of the management of high-risk population. Stroke vascular neurology. 2020;5(3):270-8.

5. Lei Z, Adrian W, Yishan L, Wenyan L, C CWW, M AJ, Vincent LLRK, Lin M. S: The Additional Contribution of White Matter Hyperintensity Location to Post-stroke Cognitive Impairment: Insights From a Multiple-Lesion Symptom Mapping Study. Frontiers in neuroscience 2018, 12.

6. Ma L. Status of diagnosis and treatment of cognitive impairment after stroke. Chinese Journal of Senile Cardio-Cerebrovascular disease 2020(No.4 volume):337-339.

7. Leo V, Sihvonen A, Linnavalli T, Tervaniemi M, Laine M, Soinila S, Särkämö T. Cognitive and neural mechanisms underlying the mnemonic effect of songs after stroke. Neurolmage Clinical. 2019;24:101948.

8. Yin M, Liu Y, Zhang L, Zheng H, Peng L, Ai Y, Luo J, Hu X. Effects of rTMS Treatment on Cognitive Impairment and Resting-State Brain Activity in Stroke Patients: A Randomized Clinical Trial. Front Neural Circuits. 2020;14:563777.

9. Shaker H, Sawan S, Fahmy E, Ismail R, Elrahman S. Effect of transcranial direct current stimulation on cognitive function in stroke patients. The Egyptian journal of neurology psychiatry neurosurgery. 2018;54(1):32.

10. Merriman N, Gillan D, Pender N, Williams D, Horgan F, Sexton E, Johnston M, Bennett K, Donnelly N, Wren M, et al: The StrokeCog study: development and description of a cognition-focused 
psychological intervention to address cognitive impairment following stroke. Psychology \& health 2020:1-18.

11. Shih C, Yeh C, Yang J, Lane H, Huang C, Lin J, Chen T, Liao C. Reduced use of emergency care and hospitalization in patients with post-stroke cognitive impairment treated with traditional Chinese medicine. QJM: monthly journal of the Association of Physicians. 2019;112(6):437-42.

12. Chen L, Fang J, Ma R, Gu X, Chen L, Li J, Xu S. Additional effects of acupuncture on early comprehensive rehabilitation in patients with mild to moderate acute ischemic stroke: a multicenter randomized controlled trial. BMC Complement Altern Med. 2016;16:226.

13. Su Y, Yuki M, Otsuki M. Non-Pharmacological Interventions for Post-Stroke Fatigue: Systematic Review and Network Meta-Analysis. Journal of clinical medicine 2020, 9(3).

14. Shao Y, Wang P, Wang Q, Yu L, Zhang L, Wang W. Eye-acupuncture with rehabilitation therapy for stroke. Medicine. 2020;99(18):e20096.

15. Lin R, Yu K, Li X, Tao J, Lin Y, Zhao C, Li C, Chen L. Electroacupuncture ameliorates post-stroke learning and memory through minimizing ultrastructural brain damage and inhibiting the expression of MMP-2 and MMP-9 in cerebral ischemia-reperfusion injured rats. Mol Med Rep. 2016;14(1):22533.

16. Yang F, Luo K, Yang X, Ren H, Gao F, Qian L. qi[Clinical observation of long-time needle retaining at Baihui (GV 20) on post-stroke cognitive disorder of deficiency and blood stasis]. Zhongguo zhen jiu = Chinese acupuncture moxibustion. 2018;38(11):1151-6.

17. Zhan J, Wang X, Cheng N, Tan F. [Electroacupuncture for post stroke cognitive impairment: a systematic review and Meta-analyses]. Zhongguo zhen jiu = Chinese acupuncture moxibustion. 2017;37(10):1119-25.

18. Moher D, Hopewell S, Schulz K, Montori V, Gøtzsche P, Devereaux P, Elbourne D, Egger M, Altman D. CONSORT 2010 explanation and elaboration: updated guidelines for reporting parallel group randomised trials. International journal of surgery (London England). 2012;10(1):28-55.

19. Schulz K, Altman D, Moher D. CONSORT 2010 statement: updated guidelines for reporting parallel group randomised trials. International journal of surgery (London England). 2011;9(8):672-7.

20. MacPherson H, Altman D, Hammerschlag R, Youping L, Taixiang W, White A, Moher D. Revised STandards for Reporting Interventions in Clinical Trials of Acupuncture (STRICTA): Extending the CONSORT statement. Journal of evidence-based medicine. 2010;3(3):140-55.

21. Weng LK. [A preliminary study on the revision of diagnostic criteria for apoplexy] [Master]. Guangzho Traditional Chinese Medicine University; 2011.

22. Skrobot O, Black S, Chen C, DeCarli C, Erkinjuntti T, Ford G, Kalaria R, O'Brien J, Pantoni L, Pasquier F, et al. Progress toward standardized diagnosis of vascular cognitive impairment: Guidelines from the Vascular Impairment of Cognition Classification Consensus Study. Alzheimer's dementia: the journal of the Alzheimer's Association. 2018;14(3):280-92.

23. Zhang T, Zhao J, Li X, Bai Y, Wang B, Qu Y, Li B, Zhao S. Chinese Stroke Association guidelines for clinical management of cerebrovascular disorders: executive summary and 2019 update of clinical 
management of stroke rehabilitation. Stroke vascular neurology. 2020;5(3):250-9.

24. Wang S, Yang H, Zhang J, Zhang B, Liu T, Gan L, Zheng J. Efficacy and safety assessment of acupuncture and nimodipine to treat mild cognitive impairment after cerebral infarction: a randomized controlled trial. BMC Complement Altern Med. 2016;16:361.

25. Chen X, Fang J, Shang Y, Ma R, Fang Z, Xuan L, Wu X, Bao Y, Chen S. [Acupuncture combined with western medication for mild cognitive disorder after stroke: a rando- mized controlled trial]. Zhongguo zhen jiu = Chinese acupuncture moxibustion. 2016;36(4):337-41.

26. Liu L: [Clinical observation on therapeutic effect of Yu's head acupuncture for mild cognitive dysfunction]. [Master]. Heilongjiang University of Traditional Chinese Medicine; 2017.

27. Xiong ZY. [Effects of Baduanjin exercise on motor function in patients with cognitive impairment after stroke]. [Master].Fujian Traditional Chinese Medicine University; 2018.

28. Chen A, Lin Z, Lan L, Xie G, Huang J, Lin J, Peng J, Tao J, Chen L. Electroacupuncture at the Quchi and Zusanli acupoints exerts neuroprotectiverole in cerebral ischemia-reperfusion injured rats via activation of the PI3K/Aktpathway. International Journal of Molecular Medicine 2012, 30(4).

29. Jiang HW, Ju YL, Shao W, Chen GH, Luo LJ, Wang JL, Gao Y. [Effects of early application of Huanglian Jiedu Decoction on cognitive function and Notch signaling pathway in animal models with multiple cerebral infarction]. Jilin journal of traditional chinese medicine. 2020;40(06):779-83.

30. Song Y, Zhao F, Huang WH, Chen Y. [Therapeutic effect of acupuncture combined with rehabilitation training on cognitive impairment after stroke based on the theory of "Brain and kidney correlation]. Shanghai Journal of Acupuncture. 2020;39(06):657-60.

31. Wang QF, Wang K, Wang FB, Li R, Wang JJ, Li J. [ Efficacy of Zhi three acupuncture points combined with head acupuncture in the treatment of vascular cognitive impairment after stroke and its influence on vilip-1 and IGF-1 levels]. Journal of clinical acupuncture moxibustion. 2020;36(09):5-8.

32. Zhang W, Lin RH, Li ZF, Tao J, Chen LD. [Effects of electroacupuncture (ECT) Shenting and Baihui on the learning and memory ability and the level and phosphorylation of cyclic adenosine effector element binding protein in hippocampus CA1 in rats with cerebral ischemia-reperfusion]. Theory Practice of Rehabilitation in China. 2016;22(11):1241-5.

33. Guo J, Yu HY, Gao Y, Chen D. [the effect of acupoint massage combined with rehabilitation training on stroke patients with unilateral neglect]. Chinese Journal of Rehabilitation Medicine. 2017;32(09):1012-5.

\section{Tables}

Table 1 Acupoints selected for use in the study 


\begin{tabular}{|c|c|c|}
\hline acupoints & location & Manipulation \\
\hline $\begin{array}{l}\text { Shenting(GV24) } \\
\text { (bilateral) }\end{array}$ & $\begin{array}{l}\text { On the head, the hairline up straight } \\
\text { about } 0.5 \text { inch }\end{array}$ & $\begin{array}{l}\text { Insert forward } 1 \text { inch, with at an angle } \\
\text { of } 15-30^{\circ} \text { oblique along the scalp, } \\
\text { twist for } 200 \text { times /min and keep } \\
\text { twisting for } 1 \text { min until getting Qi, } \\
\text { electric acupuncture with } \\
\text { Qianding(GV } 21) \text {, }\end{array}$ \\
\hline Benshen (GB13) & $\begin{array}{l}\text { On the head, } 0.5 \text { inch above the front } \\
\text { hairline, } 3 \text { inch beside the } \\
\text { Shenting(GV24), the intersection point } \\
\text { between the inner } 2 / 3 \text { and outer } 1 / 3 \text { of } \\
\text { the connection line between the } \\
\text { Shenting(GV24) and Tou Wei(ST8) }\end{array}$ & $\begin{array}{l}\text { Insert forward } 1 \text { inch, with at an angle } \\
\text { of } 15-30^{\circ} \text { oblique along the } \\
\text { scalp, twist for } 200 \text { times /min and } \\
\text { keep twisting for } 1 \mathrm{~min} \text { until getting } \\
\text { Qi }\end{array}$ \\
\hline Baihui(GV20) & $\begin{array}{l}\text { On the head, the intersection of the } \\
\text { midline and the line tips of the ears }\end{array}$ & $\begin{array}{l}\text { Insert forward } 1 \text { inch, with at an angle } \\
\text { of } 15-30^{\circ} \text { oblique along the } \\
\text { scalp, twist for } 200 \text { times /min and } \\
\text { keep twisting for } 1 \text { min until getting } \\
\text { Qi, electric acupuncture with } \\
\text { Houshencong }\end{array}$ \\
\hline $\begin{array}{l}\text { Sishencong(EX- } \\
\text { HN1) }\end{array}$ & $\begin{array}{l}\text { On the head, conclude four } \\
\text { acupionts(Qianshencong, } \\
\text { Houshencong, Zuoshencong and } \\
\text { Youshencong), which } 1 \text { inch beside } \\
\text { Baihui on the four directions(front, } \\
\text { back, left and right) }\end{array}$ & $\begin{array}{l}\text { Insert toward Baihui } 1 \text { inch, with at an } \\
\text { angle of } 15-30^{\circ} \text { oblique along the } \\
\text { scalp, twist for } 200 \text { times /min and } \\
\text { keep twisting for } 1 \text { min until getting } \\
\text { Qi. Houshencong is used electric } \\
\text { acupuncture with Baihui }\end{array}$ \\
\hline $\begin{array}{l}\text { Fengchi(GB-20)( } \\
\text { bilateral) }\end{array}$ & $\begin{array}{l}\text { On the nape, below the occipital, on a } \\
\text { level with Fengfu (DU-16), in the } \\
\text { depression between the upper portion } \\
\text { of trapezius and the } \\
\text { sternocleidomastoid }\end{array}$ & $\begin{array}{l}\text { Normal manipulation, electric } \\
\text { acupuncture will be use between left } \\
\text { Fengchi and Zuoshencong, right } \\
\text { Fengchi and Youshencong }\end{array}$ \\
\hline $\begin{array}{l}\text { Fenglong (ST40) } \\
\text { (bilateral) }\end{array}$ & $\begin{array}{l}\text { On the crus anterolateral, 8inch above } \\
\text { the tip of the lateral malleolus }\end{array}$ & Normal manipulation \\
\hline $\begin{array}{l}\text { Zusanli(ST36) } \\
\text { (bilateral) }\end{array}$ & $\begin{array}{l}\text { On the crus anterolateral, 3inch below } \\
\text { the knee and } 1 \text { inch beside the tibial } \\
\text { crest. }\end{array}$ & Normal manipulation \\
\hline $\begin{array}{l}\text { Xuanzhong(GB39) } \\
\text { (bilateral) }\end{array}$ & $\begin{array}{l}\text { On the crus anterolateral, 3inch above } \\
\text { the tip of the lateral malleolus }\end{array}$ & Normal manipulation \\
\hline Top midline & $\begin{array}{l}\text { On the top of the head, the line } \\
\text { between the Baihui and Qianding }\end{array}$ & $\begin{array}{l}\text { Insert forward } 1 \text { inch, with at an angle } \\
\text { of } 15-30^{\circ} \text { oblique along the scalp, } \\
\text { twist for } 200 \text { times /min and keep } \\
\text { twisting for } 1 \text { min until getting Qi }\end{array}$ \\
\hline Forehead midline & $\begin{array}{l}\text { In the middle of the forehead, above } \\
\text { and down } 0.5 \text { inch of front hairline }\end{array}$ & $\begin{array}{l}\text { Insert forward } 1 \text { inch, with at an angle } \\
\text { of } 15-30^{\circ} \text { oblique along the scalp, } \\
\text { twist for } 200 \text { times /min and keep } \\
\text { twisting for } 1 \mathrm{~min} \text { until getting Qi }\end{array}$ \\
\hline $\begin{array}{l}\text { No. } 1 \text { line near the } \\
\text { top (bilateral) }\end{array}$ & $\begin{array}{l}\text { On the top of the head, } 1.5 \text { left and } \\
\text { right sides beside the Du meridian }\end{array}$ & $\begin{array}{l}\text { Insert forward } 1 \text { inch, with at an angle } \\
\text { of } 15-30^{\circ} \text { oblique along the scalp, }\end{array}$ \\
\hline
\end{tabular}

Page 15/18 
Table 2 Outcome measurements at each timepoint

\begin{tabular}{|lllllll|}
\hline Measurements & Baseline & & Treatment phase & \multicolumn{2}{c|}{ Follow-up phase } \\
\cline { 2 - 6 } & -1 weeks & Oweek & 8weeks & 12 week & 16weeks & 24 weeks \\
MoCA & $\times$ & $\times$ & $\times$ & $\times$ & $\times$ & $\times$ \\
MMSE & $\times$ & $\times$ & $\times$ & $\times$ & $\times$ & $\times$ \\
ADL & & $\times$ & & $\times$ & $\times$ & $\times$ \\
HAMD & $\times$ & $\times$ & & $\times$ & $\times$ & $\times$ \\
RS-fMRI & $\times$ & & $\times$ & & \\
fNIRS & $\times$ & & $\times$ & \\
\hline
\end{tabular}

\section{Figures}




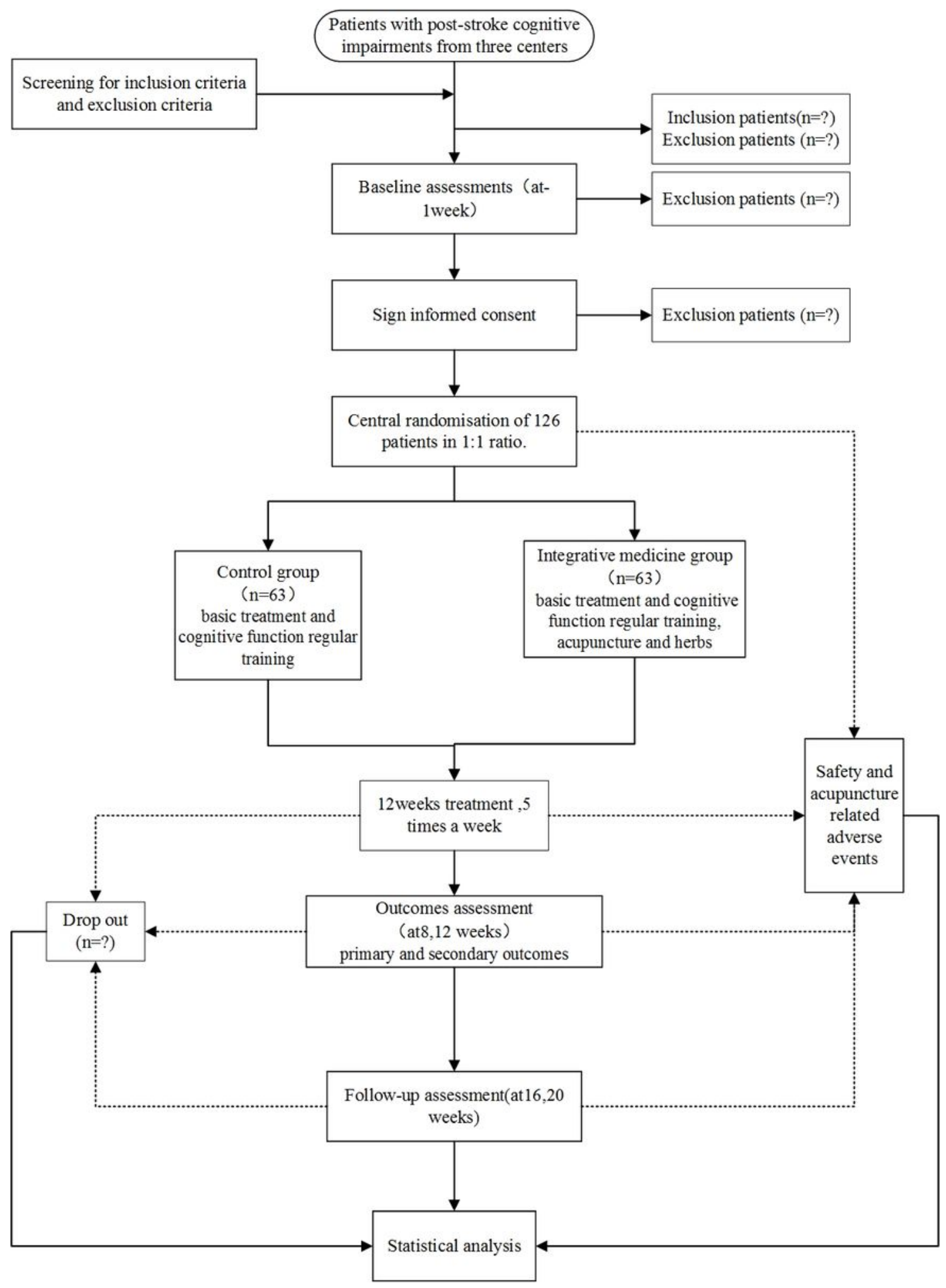

Figure 1

Fig 1 shows the trial design.

\section{Supplementary Files}

This is a list of supplementary files associated with this preprint. Click to download. 
- SPIRITChecklistdownload8Jan13.doc 